\title{
A Molecular Docking Study towards Finding Herbal Treatment against Polycystic Ovary Syndrome (PCOS)
}

\author{
Anshul Kamboj, Devvret Verma, Damini Sharma, Kumud Pant, Bhasker Pant, Vijay Kumar
}

\begin{abstract}
Polycystic ovary syndrome (PCOS) is one of the which has affected reproductive-age women, it is characterized by hyperinsulinemia, hyperandrogenism, menstrual irregularities, and long-term metabolic disturbances. CYP 17 (P450c 17a) is an enzyme that plays an essential role in the biosynthesis of adrenal and gonadal steroids. Due to overexpression of the CYP17 encoding gene androgen is converted more efficiently to testosterone causing hyperandrogenism. By inhibiting this enzyme activity androgen synthesis can be prevented in the ovary. In this study, virtual screening of the phytochemicals of fruit from plant Terminalia chebula, Terminaliabellirica and Emblica officinalis were used as a ligand to identify a potent inhibitor of CYP17 enzyme. The binding affinity of phytochemicals with the target protein CYP17 with the aid of AutoDockVina were explored. Metformin, spironolactone and clomiphene were used as control and binding energy of phytochemicals was compared with the docking score of control. All the phytocompounds shows inhibition of the CYP17 enzyme with a docking score of $\mathbf{- 3 . 7}$ to -9.5 . Chebulanin, corilagin, neochebulinic acid, ellagic acid, chebulinic acid,1,6-diO-galloyl-d-glucose, 3,4,6-tri-O-glloyl-d-glucose, terchebulin, terflavin A, maslinic acid, arjunin, Isoquercitrin, rutin and chebulagic acid shows properties of potent inhibitor. This study reveals that the phytochemicals of fruit from plant Terminalia chebula, T.bellirica and Emblica officinalis can be used as a potential de novo drug to treat infertility in PCOS.
\end{abstract}

Keywords: Emblica officinalis, arjunin, Isoquercitrin, Terminalia, Chebulanin.

\section{INTRODUCTION}

Polycystic ovary syndrome (PCOS) is the commonest endocrinological disorder affecting 5-10\% of reproductive age women around the world. It is characterized by hyperinsulinemia, hyperandrogenism, menstrual irregularities, and long-term metabolic disturbances in the females(Kamel, 2013; Amudha and Rani, 2016).In this disease hormonal imbalance affects the growth of follicle during the ovarian cycle and cause the affected follicle to remain in the ovary.

Revised Manuscript Received on September 25, 2019.

Anshul Kamboj, Department of Biotechnology, Graphic Era Deemed to be University, Dehradun, India.

Devvret Verma, Department of Biotechnology, Graphic Era Deemed to be University, Dehradun, India.

Damini Sharma, Department of Biotechnology, Graphic Era Deemed to be University, Dehradun, India.

Kumud Pant, Department of Biotechnology, Graphic Era Deemed to be University, Dehradun, India.

Bhasker Pant, Professor, Department of Computer Science and Engineering, Graphic Era Deemed to be University, Dehradun, India.

Vijay Kumar, Professor, Department of Physics, Graphic Era Hill University, Dehradun, India.
Women with PCOS usually experience diabetes, metabolic syndrome and obesity which are associated with increased risk of infertility (Apridonidzeet al., 2005). Syndromes related to ovaries can be due to sudden elevation in hormones, insulin or glucose levels which are pivotal in implantation as well as the development of the embryo. Abnormal insulin levels lead to poor egg quality which makes the inception of pregnancy difficult whereas elevated luteinizing hormones can decrease the chance of conception and leads to miscarriage. In most of the PCOS cases the main symptom is increased level of androgen. The promoter region of enzyme CYP 17 encodes a specific androgen regulating protein $\mathrm{P} 45017 \alpha$ hydroxilase, which plays an essential role in reproductive function and obesity (Amudha and Rani, 2016).

CYP17 is a microsomal enzyme and is essential for the biosynthesis of adrenal and gonadal steriods. It functions as hydroxylase and lyase. It is expressed in tissues zona recticulars and zona fasciculata of the adrenal cortex as well as gonadal tissues. CYP17 alpha is involved in the formation of four of the main steroid hormones, namely, testosterone, estradiol, cortisol, and DEHA. During the enzymatic activity, it first acts upon the pregnenolone and progesterone at the $\mathrm{C} 17$ position causing hydroxylation and form 17-hydroxypregnenolone and 17-hydroxyprogesterone and then it cleaves $\mathrm{C} 17-\mathrm{C} 20$ bond of 17hydroxypregnenolone and 17-hydroxyprogesteron to form dehydroepiandrosterone and androstenedione(Akhtaret al., 2005).In PCOS due to dysregulation of steroidogenesis within the ovaries and adrenal glands hypoandrogenism occurs. Due to overexpression of CYP17 encoding gene androgen is converted more efficiently to testosterone (Yoshimotoet al., 2016).

Various synthetic drugs such as metformin, spironolactone and clomiphene are used to treat this disorder but these synthetic drugs cause side effects such as congenital heart disease. Thus, natural compounds are used to treat the infertility which does not have any side effects. The phytochemicals of fruit from plant Terminalia chebula,Terminaliabellirica and Emblica officinalis can be used as a potent inhibition of CYP17 enzyme(Chouhanet al., 2013). In this study, we have explored the binding affinity of phytochemicals with the target protein CYP17.

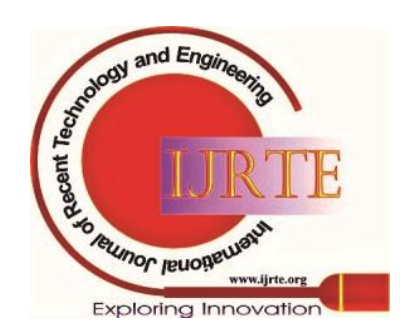




\section{MATERIALS AND METHODS}

\section{A. Protein preparation}

The raw X-ray crystal structure of enzyme CYP17 (PDB ID: 3RUK)was retrieved from Research Collaboratory for Structural Bioinformatics Protein Data Bank( https://www.rcsb.org/)(Bermanet al., 2000). Structure was prepared by removing existing ligands and removing water molecules by PyMOLv2.3.3, it is comprehensive molecular visualization software which enables users to observe the 3D structures of the compounds (Schrödinger,2020).

\section{B. Ligand preparation:}

The 3D structures of phytochemicals were retrieved from the PubChem database(www.pubchem.ncbi.nlm.nih.gov)(Butkiewiczet al., 2013).The ligands were further converted into .pdbqt format using open babel wizard of PyRx (Dallakyanet al., 2015).

\section{Virtual screening and docking:}

Molecule binding affinity was predicted from docking analysis. Protein-ligand docking was done with the help of PyRx. A virtual screening software for in silico drug discovery which is used to screen compound against a potential drug target (Dallakyanet al., 2015).

\section{RESULT AND DISCUSSION}

PCOS is caused due to cysts formation in the ovary and affects the ovarian cycle of reproductive-aged women around the age of 12-51. Drug Metformin, Spironolactone, and clomiphene are some drugs used to treat this disease. Metaformin decreased the hepatic glucose production, intestinal absorption of glucose and improve sensitivity by increasing glucose uptake and utilization. Spironolactone is a corrival of aldosterone, it binds to receptors in the distal renal tubule and creates an antiandrogen effect via competition for the androgen receptor, hence, suppresses the cytochrome P450 and decreases steroidogenesis (Akhtar et al., 2005)
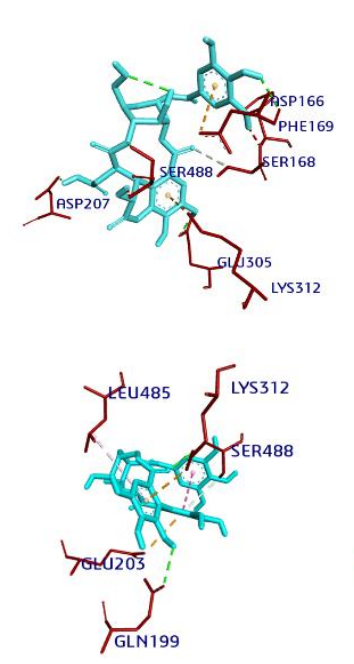

a. 3RUK_Chebulanin

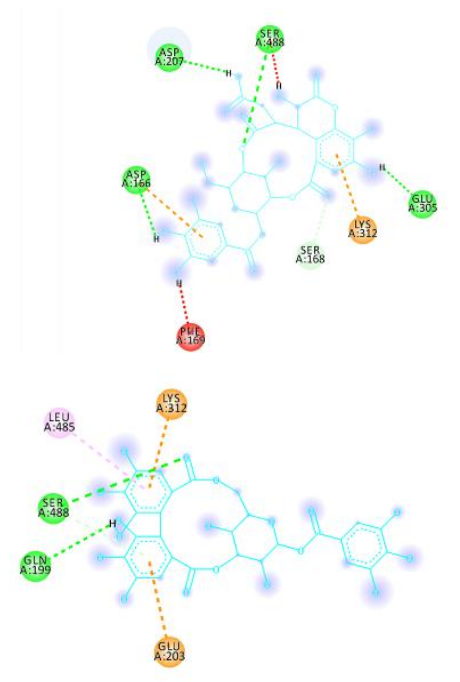

b. 3RUK_Corilagin
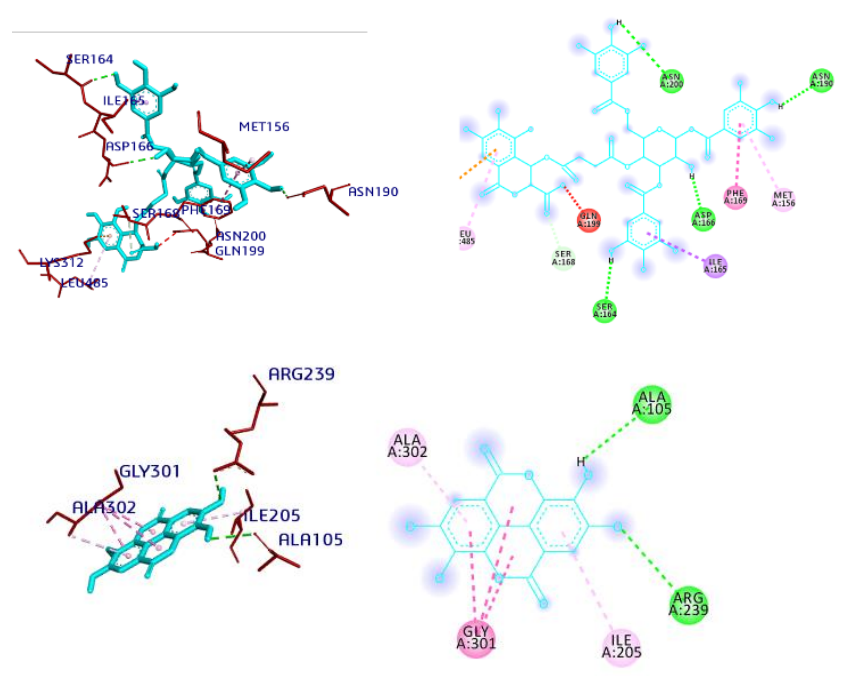

c. 3RUK_Neochebulinic acid d. 3RUK_Ellagic acid
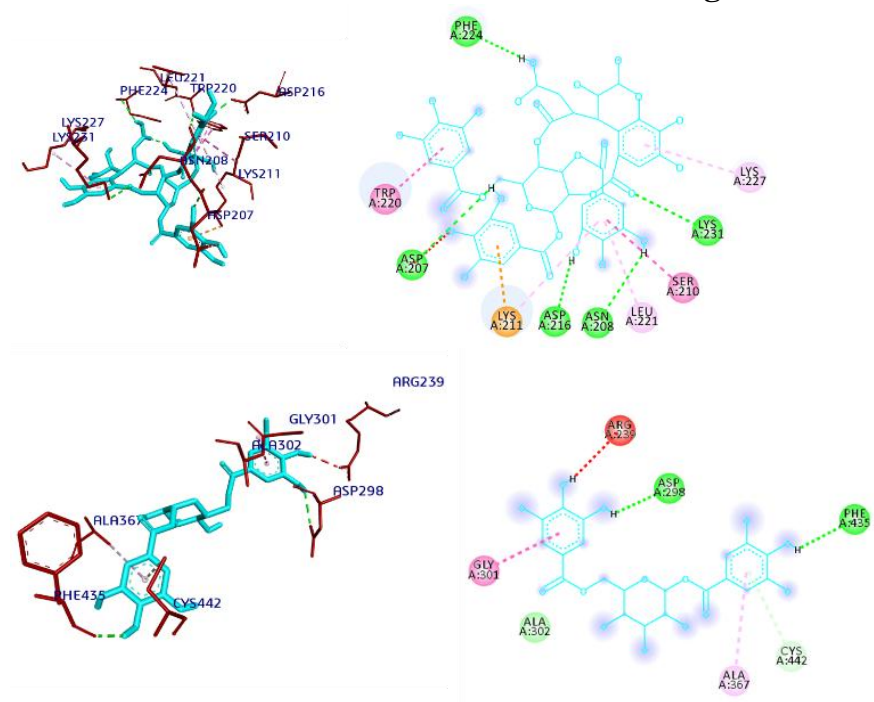
e. 3RUK_Chebulinic acid f. 3RUK_1,6-di-O-galloyl- d-glucose
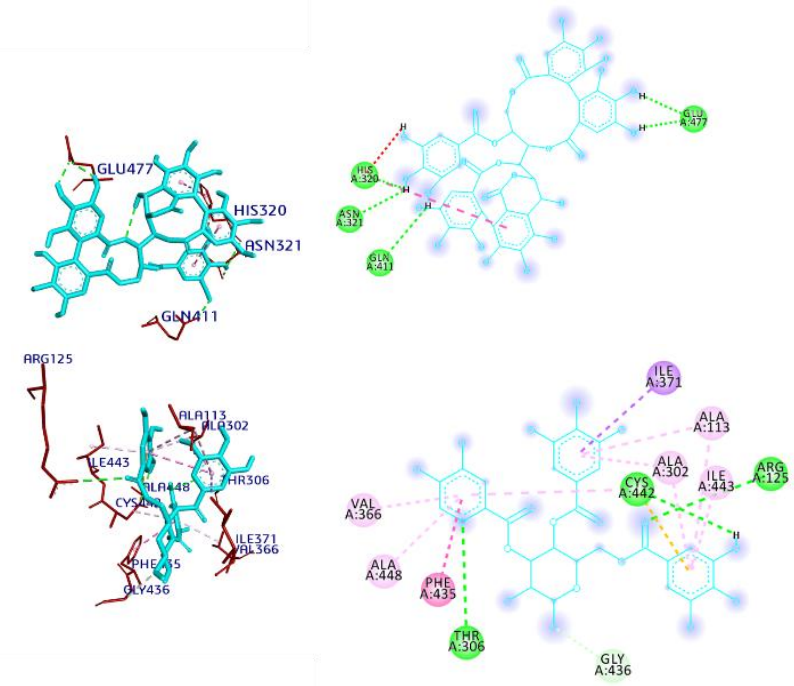

g. 3RUK_Casuarinin

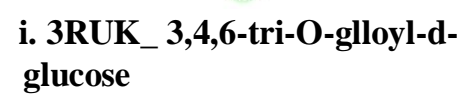
glucose

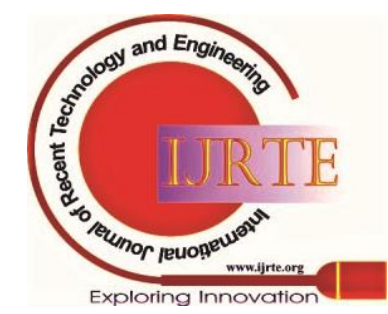



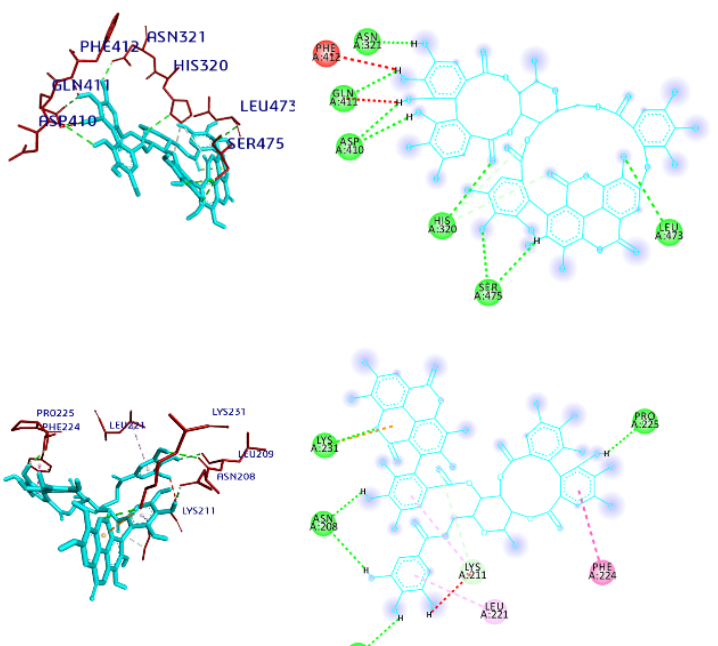

(58)

j. 3RUK_Terchebulin k. 3RUK_Terflavin A
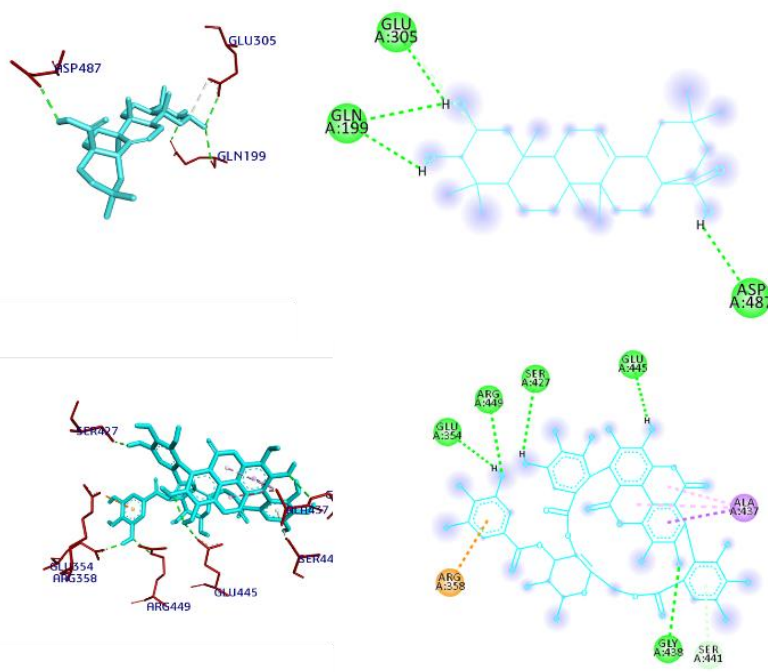

1. 3RUK_Maslinic acid

m.3RUK_Arjunin
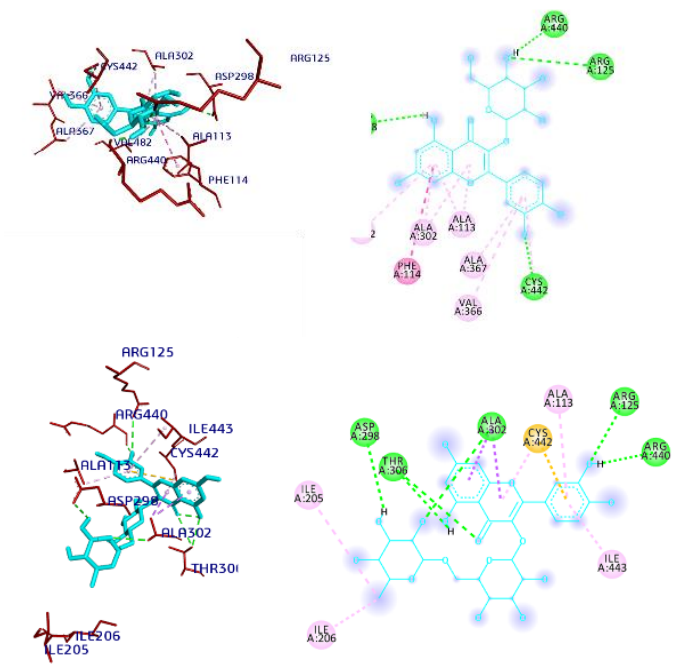

n. 3RUK_Isoquercitrin o. 3RUK_Rutin
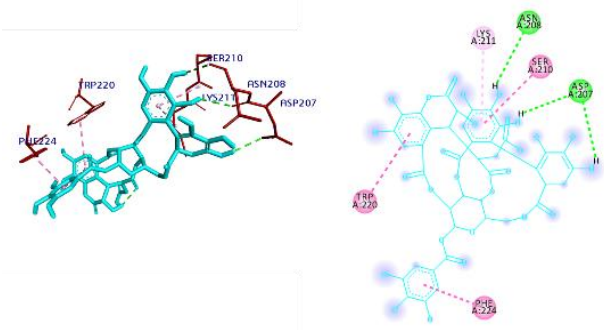

p. 3RUK_Chebulagic acid

Figure 1 (a-p): The docked poses of the protein with the phytochemicals

Clomiphene,Metformin and Spironolactone are FDA approved drug used for treating infertility. In the current study, these drugs were taken as a control. Structure of target protein Cyp17 was retrieved from the RCSB and structure of phytochemicals and drugs were retrieved from the PubChem. The target protein, PDBID:3RUKwas docked withcontrol drugs and the phytochemicals of the studied plants. The more negative value of the docking score represents better docking affinity and the docking of ligand and protein were ranked based on binding affinity. Molecular docking was performed with phytocompounds and a total of 34 phytocompounds were docked with the target protein CYP17. Treatment of PCOS is done with the help of synthetic drugs which cause problems such as congenital heart disease. Therefore, there is a need for alternative treatment which do not or have fewer side effects. Ayurveda and natural compounds had played a potent role in curing diseases in the past and can be used in present to cure a wide range of diseases. Hence natural compounds can be used as an alternative to the present medication (Pandeyet al., 2013). In the current study, the phytochemicalshave been screenedas a possible inhibitor of CYP 17 enzymewhich is responsible for the biosynthesis of adrenal and gonadal steriods. Chebulanin, corilagin, neochebulinic acid, ellagic acid, chebulinic acid,1,6-di- $O$ galloyl-d-glucose, 3,4,6-tri- $O$-glloyl-d-glucose, terchebulin, terflavin A, maslinic acid, arjunin, Isoquercitrin,rutin and chebulagic acid shows better binding affinities with the target protein and hence act as potent inhibitors of CYP17enzyme(Table1, Figure 1 a-p).

Table1. Binding energy of phytochemicals with target proteins

\begin{tabular}{|c|c|c|}
\hline $\begin{array}{c}\text { PHYTOCHEMICAL } \\
\text { NAME }\end{array}$ & CID & $\begin{array}{l}\text { Protein } \\
\text { binding } \\
\text { affinity }\end{array}$ \\
\hline \multicolumn{3}{|c|}{ Terminalia chebula } \\
\hline chebulanin & 75034370 & -8.1 \\
\hline corilagin & 73568 & -8.1 \\
\hline neochebulinic acid & 44584488 & -8.6 \\
\hline ellagic acid & 5281855 & -8 \\
\hline chebulinic acid & 72284 & -8.2 \\
\hline 1,6-di-O-galloyl-d-glucose & 91227631 & -8.3 \\
\hline casuarinin & 157395 & -8.2 \\
\hline 3,4,6-tri- $O$-glloyl-d-glucose & 14188641 & -9.2 \\
\hline terchebulin & 16175789 & -8.5 \\
\hline
\end{tabular}




\begin{tabular}{|l|l|l|}
\hline terflavin A & 16175788 & -8.2 \\
\hline maslinic acid & 73659 & -8 \\
\hline succinic acid & 1110 & -4.1 \\
\hline betasitosterol & 86821 & -7.5 \\
\hline shikimic acid & 8742 & -5.4 \\
\hline phloroglucinol & 359 & -4.4 \\
\hline pyrogallol & 1057 & -4.8 \\
\hline palmitic acid & 985 & -5.2 \\
\hline linoleic acid & 5280450 & -5.1 \\
\hline Oleic acid & 445639 & -5.8 \\
\hline chebulosides II & 102005082 & -7.6 \\
\hline arjunin & 102316370 & -8.3 \\
\hline arjunglucoside & 14658050 & -7.6 \\
\hline behenic acid & 8215 & -4.6 \\
\hline ethanedioic acid & 971 & -3.7 \\
\hline eugenol & 3314 & -5.4 \\
\hline Isoquercitrin & 5280804 & -8.1 \\
\hline \multicolumn{2}{|l|}{} \\
\hline isoguaiacin & 10314441 & -6.9 \\
\hline termilignan & 466076 & -7.3 \\
\hline thannilignan & 466077 & -6.9 \\
\hline anolignan (B), & 72388 & -6.9 \\
\hline Emblica officinalis & 54670067 & -5.2 \\
\hline vitamin c & 13250 & -5.5 \\
\hline phyllemblin & 5280805 & -9.5 \\
\hline rutin & 442674 & -8.2 \\
\hline Chebulagic acid & & \\
\hline \multicolumn{1}{|l|}{ Control drugs binding affinity with target protein } \\
\hline Metformin & & -5.8 \\
\hline Spironolactone & -7.5 \\
\hline Clomiphene & -6.8 \\
\hline \multicolumn{2}{|l|}{} \\
\hline
\end{tabular}

\section{CONCLUSION}

In the drug discovery, screening plays a crucial role as it saves costs as well as time. In this study, molecular docking has been used to examine the binding affinity of compounds and CYP17. Phytocompounds such as chebulanin, corilagin, neochebulinic acid, ellagic acid, chebulinic acid,1,6-di- $O$ galloyl-d-glucose, 3,4,6-tri- $O$-glloyl-d-glucose, terchebulin, terflavin A, maslinic acid, arjunin, Isoquercitrin, rutin, chebulagic acid have good pose with the protein. Potential fertility drugs can be made from the natural compounds of Terminalia chebula,t.bellirica and emblica officinalis and can be used to treat PCOS effectively.

\section{REFRENCES}

1. Kamel, H. H. (2013). Role of phyto-oestrogens in ovulation induction in women with polycystic ovarian syndrome. European Journal of Obstetrics \&Gynecology and Reproductive Biology, 168(1), 60-63.

2. Amudha, M., \& Rani, S. (2016). In silico molecular docking studies on the phytoconstituents of cadabafruticosa (1.) Druce for its fertility activity. Asian J Pharm Clin Res, 9(2), 48-50.

3. Apridonidze, T., Essah, P. A., Iuorno, M. J., \&Nestler, J. E. (2005). Prevalence and characteristics of the metabolic syndrome in women with polycystic ovary syndrome. The Journal of Clinical Endocrinology \& Metabolism, 90(4), 1929-1935.

4. Akhtar, M. K., Kelly, S. L., \&Kaderbhai, M. A. (2005). Cytochrome b5 modulation of $17 \alpha$ hydroxylase and 17-20 lyase (CYP17) activities in steroidogenesis. Journal of Endocrinology, 187(2), $267-$ 274 .
5. Yoshimoto, F. K., Gonzalez, E., Auchus, R. J., \&Guengerich, F. P. (2016). Mechanism of $17 \alpha, 20$-Lyase and New Hydroxylation Reactions of Human Cytochrome P450 17A1 18O LABELING AND OXYGEN SURROGATE EVIDENCE FOR A ROLE OF A PERFERRYL OXYGEN. Journal of Biological Chemistry, 291(33), 17143-17164.

6. Chouhan, B., Kumawat, R. C., Kotecha, M., Ramamurthy, A., \&Nathani, S. (2013). Triphala: A comprehensive ayurvedic review. Int J Res Ayurveda Pharm, 4(4), 612-617.

7. Berman, H. M., Westbrook, J., Feng, Z., Gilliland, G., Bhat, T. N., Weissig, H., .. \& Bourne, P. E. (2000). The protein data bank. Nucleic acids research, 28(1), 235-242.

8. The PyMOL Molecular Graphics System, Version 1.2r3pre, Schrödinger, LLC. https://pymol.org/2/

9. Butkiewicz, M., Lowe, E. W., Mueller, R., Mendenhall, J. L., Teixeira, P. L., Weaver, C. D., \&Meiler, J. (2013). Benchmarking ligand-based virtual High-Throughput Screening with the PubChem database. Molecules, 18(1), 735-756.

10. Dallakyan, S., \& Olson, A. J. (2015). Small-molecule library screening by docking with PyRx. In Chemical biology (pp. 243-250). Humana Press, New York, NY.

11. Pandey, M. M., Rastogi, S., \& Rawat, A. K. S. (2013). Indian traditional ayurvedic system of medicine and nutritional supplementation. Evidence-Based Complementary and Alternative Medicine, 2013. 